Phys. Rev. C (2001) in press.

\title{
Proton Differential Elliptic Flow and the Isospin-Dependence of the Nuclear Equation of State
}

\author{
Bao-An Li ${ }^{*}$, Andrew T. Sustich ${ }^{\dagger}$ and Bin Zhang ${ }^{\ddagger}$ \\ Department of Chemistry and Physics \\ P.O. Box 419, Arkansas State University \\ State University, Arkansas 72467-0419, USA
}

\begin{abstract}
Within an isospin-dependent transport model for nuclear reactions involving neutron-rich nuclei, we study the first-order direct transverse flow of protons and their second-order differential elliptic flow as a function of transverse momentum. It is found that the differential elliptic flow of mid-rapidity protons, especially at high transverse momenta, is much more sensitive to the isospin dependence of the nuclear equation of state than the direct flow. Origins of these different sensitivities and their implications to the experimental determination of the isospin dependence of the nuclear equation of state by using neutron-rich heavy-ion collisions at intermediate energies are discussed
\end{abstract}

PACS numbers: 25.70.-z, 25.75.Ld., 24.10.Lx

\footnotetext{
*email: Bali@astate.edu

$\dagger^{\dagger}$ email: Sustich@astate.edu

łemail: Bzhang@astate.edu
} 
The isospin-dependence of the nuclear equation of state (EOS) is one of the most important but very poorly known property of neutron-rich matter [1]. Its determination in laboratory-controlled experiments has profound implications to the study of the structure and evolution of many astrophysical objects [2]. Nuclear reactions induced by stable neutronrich nuclei and/or radioactive beams provide a means to extract useful information about the isospin-dependence of the nuclear EOS and to explore novel phenomena in nuclear matter at extreme isospin asymmetries. A number of dedicated experiments to study the isospin dependence of the nuclear EOS have been performed/planned at several available radioactive beam facilities and the future Rare Isotope Accelerator (RIA) [3]. For these experiments to be fruitful it is important to first understand well the role of the isospin degree of freedom in nuclear reaction dynamics. Moreover, theoretical predictions on the sensitivity of experimental observables to the isospin dependence of the nuclear EOS are useful for explaining available data and planning new experiments. Recently, several useful observables have been identified in neutron-rich heavy-ion collisions at intermediate energies. These include the isospin fractionation [1] [10], neutron to proton ratio of heavy residues [11] or projectile-like fragments [0,12] and the neutron-proton differential flow [13]. Most of these observables make use of the relative multiplicities and kinetic energies of mirror nuclei. Information about the isospin dependence of the nuclear EOS can also be obtained from studying free nucleons as neutrons and protons have the opposite symmetry potentials in nuclear medium. In a recent work, one of the present authors has shown that the neutron-proton differential flow is a rather useful probe of the isospin dependence of the nuclear EOS. The method utilizes constructively both the isospin fractionation and the nuclear collective flow as well as their sensitivities to the nuclear EOS. It, however, requires measuring the neutron and proton collective flow simultaneously. Experimentally, it is easier to identify charged particles and measure their momenta accurately, thus observables using charged particles only are more useful. In this work, within an isospin-dependent transport model for nuclear reactions involving neutron-rich nuclei, we explore the sensitivity of proton collective flow to the isospin dependence of the nuclear EOS in heavy-ion collisions at intermediate energies. 
We examine both the first-order transverse flow and the second-order differential elliptic flow as functions of transverse momentum. It is found that the differential elliptic flow of midrapidity protons, especially at high transverse momenta, is very sensitive to the isospin dependence of the nuclear EOS. This sensitivity is much stronger than that found in the first-order transverse collective flow around the projectile and target rapidity.

At present, nuclear many-body theories predict vastly different isospin dependence of the nuclear EOS depending on both the calculation techniques and the bare two-body and/or three-body interactions employed, see e.g., [14 17]. Various theoretical studies (e.g., [18, 19]) have shown that the energy per nucleon $e(\rho, \delta)$ in nuclear matter of density $\rho$ and isospin asymmetry parameter $\delta$ defined as

$$
\delta \equiv\left(\rho_{n}-\rho_{p}\right) /\left(\rho_{n}+\rho_{p}\right)
$$

can be approximated very well by a parabolic function

$$
e(\rho, \delta)=e(\rho, 0)+S(\rho) \cdot \delta^{2} .
$$

In the above $e(\rho, 0)$ is the EOS of isospin symmetric nuclear matter and $S(\rho)$ is the symmetry energy at density $\rho$. The EOS should also be momentum-dependent, however, for this exploratory study we shall negelect this dependence. We shall use for isospin-symmetric nuclear matter a stiff EOS with $K_{0}=380 \mathrm{MeV}$ which can reproduce the transverse flow data equally well as a momentum-dependent soft EOS with $K_{0}=210 \mathrm{MeV}$ [20,21]. This choice will not affect our conclusions since the elliptic flow has been shown to be insensitive to the momentum-dependence of the EOS in heavy-ion collisions at beam energies below about $1.5 \mathrm{GeV} /$ nucleon if $K_{0}=380 \mathrm{MeV}$ is used [22]. The form of the symmetry energy as a function of density is rather strongly model dependent [23]. Very divergent predictions on the isospin dependence of the nuclear EOS by various many-body theories have lead to vastly different forms for the $S(\rho)$. We adopt here a parameterization used by Heiselberg and Hjorth-Jensen in their studies on neutron stars [24]

$$
S(\rho)=S_{0}\left(\rho_{0}\right) \cdot u^{\gamma}
$$


where $u \equiv \rho / \rho_{0}$ is the reduced density and $S_{0}\left(\rho_{0}\right)$ is the symmetry energy at normal nuclear matter density $\rho_{0}$. The value of $S_{0}\left(\rho_{0}\right)$ is known to be in the range of about 27-36 MeV from analyzing atomic masses [25 27]. By fitting the result of variational many-body calculations by Akmal et al [15], Heiselberg and Hjorth-Jensen found the values of $S\left(\rho_{0}\right)=32 \mathrm{MeV}$ and $\gamma=0.6$. However, as shown by many other authors previously [23] and more recently by Brown [16] using other approaches, the extracted value of $\gamma$ varies widely, even its sign is undetermined. Therefore,in this work we take the $\gamma$ as a free parameter and study its influence on the experimental observables in neutron-rich heavy-ion collisions. Further, we take a constant value of $30 \mathrm{MeV}$ for $S_{0}\left(\rho_{0}\right)$ in the following study.

It is well known that the symmetry energy has a kinetic and a potential contribution

$$
S(\rho)=\frac{3}{5} e_{\mathrm{F}}^{0} u^{\frac{2}{3}}\left(2^{\frac{2}{3}}-1\right)+V_{2}
$$

where $e_{F}^{0}$ is the Fermi energy in symmetric nuclear matter at normal density and $V_{2}$ is the potential contribution. Within our transport model, the kinetic part of the symmetry energy is simulated by using different Fermi momenta for neutrons and protons according to the local Thomas-Fermi approximation. While the potential part is taken into account by using the symmetry potential. Corresponding to Eq. 目 the symmetry potential energy density is

$$
W_{a s y}=V_{2} \rho \delta^{2},
$$

and the single-particle symmetry potential $V_{\text {asy }}^{q}$ can be obtained from

$$
V_{\text {asy }}^{q}=\frac{\partial W_{a s y}}{\partial \rho_{q}}=\left(S_{0}(\gamma-1) u^{\gamma}+4.2 u^{\frac{2}{3}}\right) \delta^{2} \pm\left(S_{0} u^{\gamma}-12.7 u^{2 / 3}\right) \delta
$$

where "+" and "-" are for $q=$ neutron and $q=$ proton, respectively. For small isospin asymmetries and densities near $\rho_{0}$ the above symmetry potentials reduce to the well-known Lane potential which varies linearly with $\delta$ [28]. Shown in Fig. 1 are the symmetry potentials at $\delta=0.2$ with the $\gamma$ parameter of $0.5,1.0$ and 2.0 for neutrons (upper branch) and protons (lower branch), respectively. Generally, the repulsive/attractive symmetry potential for neutrons/protons increases with density. The isospin dependence of the nuclear EOS can be characterized by the curvature of the symmetry energy at $\rho_{0}$ [18] 


$$
\left.K_{\text {sym }} \equiv 9 \rho_{0}^{2} \frac{\partial^{2} S(\rho)}{\partial \rho^{2}}\right|_{\rho=\rho_{0}}=9 S_{0}\left(\rho_{0}\right) \cdot \gamma(\gamma-1)
$$

The slope and curvature of the symmetry potential depends strongly on the parameter $\gamma$. We consider in the following two cases with $\gamma=0.5$ and 2 , the corresponding $K_{\text {sym }}$ parameter is $-68 \mathrm{MeV}$ and $540 \mathrm{MeV}$, respectively. This choice of the parameter $\gamma$ allows us to explore a large uncertain range of the isospin dependence of the nuclear EOS.

Our study is based on the isospin-dependent Boltzmann-Uehling-Uhlenbeck (IBUU) transport model (e.g., [4, 29]). In this model protons and neutrons are initialized in coordinate space according to their density distributions predicted by the relativistic mean-field $(\mathrm{RMF})$ theory [30,31]. In momentum space they are initialized in Fermi spheres with individual neutron or proton Fermi momentum dertermined by its local density according to the Thomas-Fermi approximation. The isospin-dependent reaction dynamics is included through isospin-dependent nucleon-nucleon scatterings and Pauli blockings, the symmetry potential $V_{\text {asy }}$ and the Coulomb potential $V_{c}^{p}$ for protons. A Skyrme-type parameterization is used for the isoscalar potential. For a review of the model, we refer the reader to ref. [5]. However, a few comments relevant to the present study are necessary here. We did not include in the EOS the curvature term which is important for reproducing surface properties of static nuclei and keeping them stable. This is because we can not find a universal coefficient of this term that can reproduce the neutron and proton density distribtions of all nuclei up to mass 200. Instead, we used the predictions by the RMF theory to initialize nucleons. It would be ideal if one can use the same model with the same interaction to first reproduce perfectly the initial neutron and proton density and momentum distributions, in particular, the neutron skins of heavy nuclei, then study the reactions between them. What we have done sofar is thus a compromise. Nevertheless, the best available knowledge about the inital neutron and proton distributions is used, and we found that the initial distributions for stable nuclei are kept stable upto about $150 \mathrm{fm} / \mathrm{c}$ with energetic particle emissions less than about 1 percent in our approach. Thus the distributions are sufficiently stable over a time period that is long enough for studying the collective flow which is mainly generated 
in the early stage of the reaction.

First, to understand the role of the symmetry potential in the reaction dynamics we have studied the central density as a function of time for the reaction of $\mathrm{Sn}+\mathrm{Sn}$. To the first order of approximation, there is essentially no difference in the evolution of the central density with $\gamma=0.5$ and 2 since the symmetry potential is rather small compared to the isoscalar nuclear potential. We have also investigated the relative roles of the symmstry and Coulomb potentials in the reaction dynamics by turning on or off one of them. We found that the Coulomb potential dominates over the attractive symmetry potential for protons for the reaction considered here. However, without including the symmetry potential the Coulomb potential along will lead artifically to more proton emissions than neutrons in the early stage of the reaction. Moreover, stable nuclei can not be kept stable using only the Coulomb potential without the symmetry potential in the transport model. We thus either turn on or off simultaneously both the Coulomb and symmetry potential in our following studies. To evaluate the degree of isospin asymmetry obtained and its correlation with density we show in Fig. 2 a scatter plot of the local density $\rho($ cell $) / \rho_{0}$ and the isospin asymmetry $\delta($ cell) of all non-vacant cells of $1 \mathrm{fm}^{3}$ volume each. The plot is made by using the parameter $\gamma=0.5$ for the $\mathrm{Sn}+\mathrm{Sn}$ reaction at the time instant of $30 \mathrm{fm} / \mathrm{c}$ when the central density is close to its maximum. It is seen that the high density region centers around the isospin asymmetry of the reaction system of $\delta_{0} \approx 0.19$ as one would expect. Cells with lower densities extends in $\delta$ toward the neutron-rich side far away from $\delta_{0}$ due to both the neutron-skins of the colliding nuclei and the isospin fractionation during the reaction. The latter is an unequal partitioning of the neutron to proton ratio $N / Z$ of asymmetric nuclear matter between low and high density regions. It is energetically favorable for the asymmetric nuclear matter to be separated into a neutron-rich low density phase and an isospin-symmetric high density one 32 36]. Since the symmetry potential is an increasing function of $\delta$, the symmetry potentials at densities less than $\rho_{0}$ but with larger isospin asymmetries are also appreciable as in the higher density region around the $\delta_{0}$. Although this symmetry potential itself has almost no effect on the compression and expansion of the central region, it does have a 
significant effect on the emission of particles and their kinetic energy spectra. As seen from Fig. 1, at densities below about $\rho_{0}$, the attractive symmetry potential for protons is stronger with the parameter $\gamma=0.5$ than $\gamma=2.0$. As a result, more protons are expected to become unbound with $\gamma=2$. Shown in Fig. 3 are the unbound proton rapidity distributions at the freeze-out for the $\mathrm{Sn}+\mathrm{Sn}$ reaction. The unbound protons are identified as those with local densities less than $\rho_{0} / 8$ and the freeze-out time is found to be about $100 \mathrm{fm} / \mathrm{c}$ for the reaction considered here. Appreciable effects of the symmetry potential on the rapidity distribution of free protons are seen over the whole rapidity range. Significantly more particles are emitted with $\gamma=2$., especially around the midrapidity. This observation is consistent with the total and differential isospin fractionation discussed in detail in refs. [4, 10, 13].

We now turn to the exploration of the isospin dependence of the nuclear EOS by studying both the first-order transverse collective flow and the second-order differential elliptic flow of unbound protons. Collective flow has been well known for its ability to reveal the pressure gradient during nuclear reactions, see e.g., 37 39]. In the local rest frame of matter the collective flow velocity $\vec{v}$, energy density $e$ and pressure $p$ are related by the relativistic Euler equation 38

$$
(e+p) \frac{\partial}{\partial t} \vec{v}=-\vec{\nabla} p
$$

The pressure gradient $\vec{\nabla} p$ plays the role of a driving force for the acceleration of the collective flow velocity $\vec{v}$. For isospin asymmetric nuclear matter the pressure $p$ has a kinetic part $p_{k i n}$, an isospin-independent interaction part $p_{0}$ and an isospin-dependent one $p_{a s y}$, i.e.,

$$
p=p_{\text {kin }}+p_{0}+p_{\text {asy }} .
$$

At temperature $T$ the kinetic contribution $p_{\text {kin }}$ is

$$
p_{\text {kin }}=T \rho\left\{1+\frac{1}{2} \sum_{n=1}^{\infty} b_{n}\left(\frac{\lambda_{T}^{3} \rho}{4}\right)^{n}\left[(1+\delta)^{1+n}+(1-\delta)^{1+n}\right]\right\},
$$

where the $b_{n}^{\prime} s$ are the inversion coefficients given in ref. [5] and

$$
\lambda_{T}=\left(\frac{2 \pi \hbar^{2}}{m T}\right)^{1 / 2}
$$


is the thermal wavelength of nucleons with mass $m$. The contribution from the isospinindependent nuclear interaction $p_{0}$ is

$$
p_{0}=\frac{1}{2} a \rho_{0}\left(\frac{\rho}{\rho_{0}}\right)^{2}+\frac{b \sigma}{1+\sigma} \rho_{0}\left(\frac{\rho}{\rho_{0}}\right)^{\sigma+1},
$$

where $a=-123.6 \mathrm{MeV}, b=70.4 \mathrm{MeV}$ and $\sigma=2$ corresponding to the stiff nuclear EOS of isospin-symmetric nuclear matter. The asymmetry pressure $p_{\text {asy }}$ due to the isospindependent potential is

$$
p_{\text {asy }}=\delta^{2}\left[S_{0}\left(\rho_{0}\right) \cdot \rho_{0} \cdot \gamma u^{\gamma+1}-8.5 u^{5 / 3}\right] .
$$

Shown in Fig. 4 is a comparison of the density derivatives of the three partial pressures. As an illustration we have selected the parameter set of $\delta=0.2, T=5 \mathrm{MeV}, \gamma=0.5$ and 2. In the whole density range reachable in heavy-ion collisions at intermediate energies, the gradient of the total pressure is overwhelmed by the contributions from the isospin-independent interaction and the kinetic pressure. The contribution from the isospin-dependent interaction is relatively small. Nevertheless, it is still useful to note that both the symmetry pressure and its density gradient increase with the $\gamma$ parameter, especially at high densities. We thus expect to see a stronger flow signal with a larger $\gamma$ parameter, but the effect should be rather small. This expectation is verified by performing the standard analysis of the transverse collective flow 40

$$
<p_{x} / A>(y)=\frac{1}{A(y)} \sum_{i=1}^{A(y)} p_{i x}(y),
$$

where $A(y)$ is the number of particles at rapidity $y$ and $p_{i x}$ is $i^{\text {th }}$ particle's transverse momentum in the reaction plane. As shown in Fig. 5., a slightly stronger transverse flow signal is obtained with $\gamma=2$ than $\gamma=0.5$ as expected. Given the difficulties and uncertainties in determining the reaction plane, the observed effect of the isospin dependence of the nuclear EOS is too small to be practically very useful. To explore the isospin-dependent EOS, one thus has to look for more delicate observables, such as the neutron-proton differential flow as proposed in ref. [13]. 
As a complementary approach which also has the advantage of not having to measure the collective flow for neutrons, we investigate the proton differential elliptic flow at midrapidity as a function of transverse momentum [41,42]

$$
<v_{2}\left(p_{t}\right)>=\frac{1}{N} \sum_{i=1}^{N} \frac{p_{i x}^{2}-p_{i y}^{2}}{p_{i t}^{2}}
$$

where $N$ is the total number of unbound protons in the rapidity range of $-0.5 \leq$ $\left(y / y_{\text {beam }}\right)_{c m s} \leq 0.5$. In this rapidity range the isospin fractionation is the strongest, i.e., the value of $N$ depends most sensitively on the parameter $\gamma$, as shown in Fig. 3. The $p_{i y}$ is the ith particle's transverse momentum perpendicular to the reaction plane. The value of $v_{2}\left(p_{t}\right)$ is thus a differential measure of the second-order flow effect as a function of transverse momentum. The sign and magnitude of $v_{2}$ reflects the result of a competition between the early "squeeze-out" ( $p_{i y}$ dominates) perpendicular to the reaction plane and the later in-plane transverse flow of nucleons. The differential elliptic flow, especially at high $p_{t}$, is expected to be more sensitive to the isospin dependence of the nuclear EOS than the first-order transverse flow. This is because all three partial pressures lead approximately to a similar difference $\delta p_{x y}^{2} \equiv p_{x}^{2}-p_{y}^{2}$ although their respective contributions to the value of $<p_{x}>$ or $\left\langle p_{y}\right\rangle$ is very different. Moreover, the early pressure created in the participant region is revealed more clearly by the value of $v_{2}\left(p_{t}\right)$ at high transverse momenta. This is due to the fact that high $p_{t}$ particles can only be produced through the most violent collisions in the early stage of the reaction. This fact is almost universal in heavy-ion collisions at all energies, see, e.g., 43 46]. These particles can only retain their high transverse momenta by escaping from the reaction zone along the direction perpendicular to the reaction plane without suffering much rescatterings.

Shown in Fig. 6 are the predicted proton differential elliptic flow as a function of transverse momentum $p_{t}$. The results presented here are obtained by using 20,000 $\mathrm{Sn}+\mathrm{Sn}$ events in each case. Below the Fermi momentum of about $0.3 \mathrm{GeV} / \mathrm{c}$ the elliptic flow increases with $p_{t}$ as predicted by the nuclear hydrodynamics [47, 48]. This is because the low $p_{t}$ particles have undergone a sufficiently large number of rescatterings to reach the hydrodynamical 
limit and to obtain an in-plane flow indicated by the positive value of $v_{2}\left(p_{t}\right)$. Particles with higher transverse momenta are mainly those emitted perpendicular to the reaction plane with negative values of $v_{2}$. These particles carry more undisturbed information about the initial high density phase of the reaction. This effect becomes stronger gradually with the increasing transverse momentum. Therefore the $v_{2}\left(p_{t}\right)$ value decreases with the increasing $p_{t}$ above the Fermi momentum. Indeed, it is seen that the elliptic flow at high $p_{t}$ is much more sensitive to the parameter $\gamma$ than the first-order transverse flow. Without the Coulomb repulsion the shadowing effect of the spectator nucleons lasts longer, it leads to a more stronger "squeez-out" and thus a more negative value of elliptic flow, especially at high $p_{t}$. As the pressure gradient increases by increasing the parameter $\gamma$, the in-plane flow becomes dominant over the "squeeze-out" in the whole range of transverse momentum. The value of $v_{2}\left(p_{t}\right)$ thus increases with the parameter $\gamma$, particular in the high $p_{t}$ region. The sensitivity observed here is much stronger than that observed in analyzing the first-order transverse flow. Although very high $p_{t}$ particles are rather rare, it is practically relatively easy for nucleons to obtain a transverse momentum upto 2 times the Fermi momentum in heavy-ion collisions at intermediate energies. In the range of $p_{t} \leq 0.5 \mathrm{GeV} / \mathrm{c}$, our results here indicate an unambiguously strong signal of the isospin dependence of the nuclear EOS far beyond the statistical error bars.

In summary, within an isospin-dependent transport model for nuclear reactions involving neutron-rich nuclei, we analyzed the first-order direct flow and the second-order differential elliptic flow for free protons in the reaction of ${ }^{124} S n+{ }^{124} S n$ at a beam energy of 50 $\mathrm{MeV} /$ nucleon and an impact parameter of $5 \mathrm{fm}$. The emphasis was placed on searching for observable signals of the isospin dependence of the nuclear EOS. We found that the differential elliptic flow, especially at high transverse of momenta, for midrapidity protons is much more sensitive to the isospin dependence of the nuclear EOS than the first-order direct flow. This finding provides a useful guide for planning future experiments to study the isospin dependence of the nuclear EOS by using neutron-rich heavy-ion collisions at intermediate energies. 
This work was supported in part by the National Science Foundation Grant No. PHY0088934, Arkansas Science and Technology Authority Grant No. 00-B-14 and Grant No. 01-B-20. 


\section{REFERENCES}

[1] Isospin Physics in Heavy-Ion Collisions at Intermediate Energies, Eds. Bao-An Li and W. Udo Schröder, Nova Science Publishers, Inc (2001, New York).

[2] J.M. Lattimer and M. Prakash, Phys. Rep. 333, 121 (2000).

[3] "Scientific Opportunties with Fast Fragmentation Beams from RIA", NSCL/MSU report, 2000, http://www.nscl.msu.edu.

[4] B.A. Li, C.M. Ko and Z. Ren, Phys. Rev. Lett. 78, 1644 (1997)

[5] B.A. Li, C.M. Ko and W. Bauer, topical review, Int. Jou. Mod. Phys. E7, 147 (1998).

[6] H. Xu et al, Phys. Rev. Lett. 85, 716 (2000).

[7] S.J. Yennello et al, Nucl. Phys. A681, 317c (2001).

[8] W. Udo Schröder et al., Nucl. Phys. A681, 418c (2001).

[9] M. Di Toro et al., Nucl. Phys. A681, 426c (2001).

[10] B.A. Li, Nucl. Phys. A681, 434c (2001).

[11] W. Tan et al., NSCL/MSU 2001 preprint, to be published.

[12] M. Veselsky et al, Phys. Rev. C62 (2000) 064613

[13] B.A. Li, Phys. Rev. Lett. 85, 4221 (2000).

[14] R.B. Wiringa, V. Fiks and A. Fabrocini, Phys. Rev. C38, 1010 (1988).

[15] A. Akmal and V.R. Pandharipande, Phys. Rev. C56, 2261 (1997); A. Akmal, V.R. Pandharipande and D.C. Ravenhall, Phys. Rev. C58, 1804 (1988).

[16] B.A. Brown, Phys. Rev. Lett. 85, 5296 (2000).

[17] C.J. Horowitz et al., Phys.Rev. C63, 025501 (2001).

[18] I. Bombaci and U. Lombardo, Phys. Rev. C44, 1892 (1991). 
[19] H. Huber, F. Weber and M..K. Weigel, Phys. Lett. B317, 485 (1993); Phys. Rev. C50, R1287 (1994).

[20] Q. Pan and P. Danielewicz, Phys. Rev. Lett. 70, 2062 (1993).

[21] J. Zhang, S. Das Gupta and C. Gale, Phys. Rev. C50, 1617 (1994).

[22] P. Danielewicz et al., Phys. Rev. Lett. 81, 2438 (1998).

[23] I. Bombaci, in Isospin Physics in Heavy-Ion Collisions at Intermediate Energies, Eds. Bao-An Li and W. Udo Schröder, Nova Science Publishers, Inc (2001, New York).

[24] H. Heiselberg and M. Hjorth-Jensen, Phys. Rep. 328, 237 (2000).

[25] M. Farine, J.M. Pearson and B. Rouben, Nucl. Phys. A304, 317 (1978).

[26] P.E. Haustein, Atomic data and nuclear data tables, 39, 185-395 (1988).

[27] J.M. Pearson et. al., Nucl. Phys. A528, 1 (1991).

[28] A.M. Lane, Nucl. Phys. 35, 676 (1962).

[29] B.A. Li and A.T. Sustich, Phys. Rev. Lett. 82, 5004 (1999).

[30] B.D. Serot and J.D. Walecka, Adv. Nucl. Phys. 16, 1 (1986).

[31] Z. Ren et al., Phys. Rev. C52, R20 (1995).

[32] H. Müller and B.D. Serot, Phys. Rev. C52, 2072 (1995).

[33] B.A. Li and C.M. Ko, Nucl. Phys. A618, 498 (1997).

[34] V. Baran, M. Colonna, M. Di Toro, and A.B. Larionov, Nucl. Phys. A632, 287 (1998).

[35] L. Shi and P. Danielewicz, Euro. Phys. Lett. 49, 34 (2000).

[36] D. Catalano, G. Giansiracusa and U. Lombardo, Nucl. Phys. A681, 390c (2001).

[37] H. Stöcker and W. Greiner, Phys. Rep. 137, 277 (1986); G.F. Bertsch and S. Das Gupta, 
Phys. Rep. 160, 189 (1988); W. Cassing, V. Metag, U. Mosel, and K. Niita, Phys. Rep. 188, 363 (1990); S. Das Gupta and G.D. Westfall, Physics Today, 46(5), 34 (1993); A. Bonasera, F. Gulminelli and J. Molitoris, Phys. Rep. 243, 1 (1994).

[38] P. Danielewicz, nucl-th/0009091 and Nucl. Phys. A (2001) in press.

[39] G.D. Westfall, Nucl. Phys. A630, 27c (1998); ibid, Nucl. Phys. A681, 343c (2001).

[40] P. Danielewicz and G. Odyniec, Phys. Lett. B157, 146 (1985).

[41] J.-Y. Ollitrault, Nucl. Phys. A638, 195c (1998) and references therein.

[42] A. Poskanzer and S.A. Voloshin, Phys. Rev. C55, 1671 (1998).

[43] B.A. Li, Nucl., Phys. A570, 797 (1994).

[44] P. Danielewicz, Phys. Rev. C51, 716 (1995).

[45] B.A. Li, B. Zhang, A. Sustich and C.M. Ko, Phys. Rev. C60, 034902 (1999).

[46] X.-N. Wang and M. Gyulassy, Phys. Rev. Lett. 86, 3496 (2001).

[47] H. Heiselberg and A. Levy, Phys. Rev. C59, 2716 (1999).

[48] P.F. Kolb, P. Huovinen, U. Heinz and H. Heiselberg, Phys. Lett. B500, 232 (2001). 


\section{FIGURES}

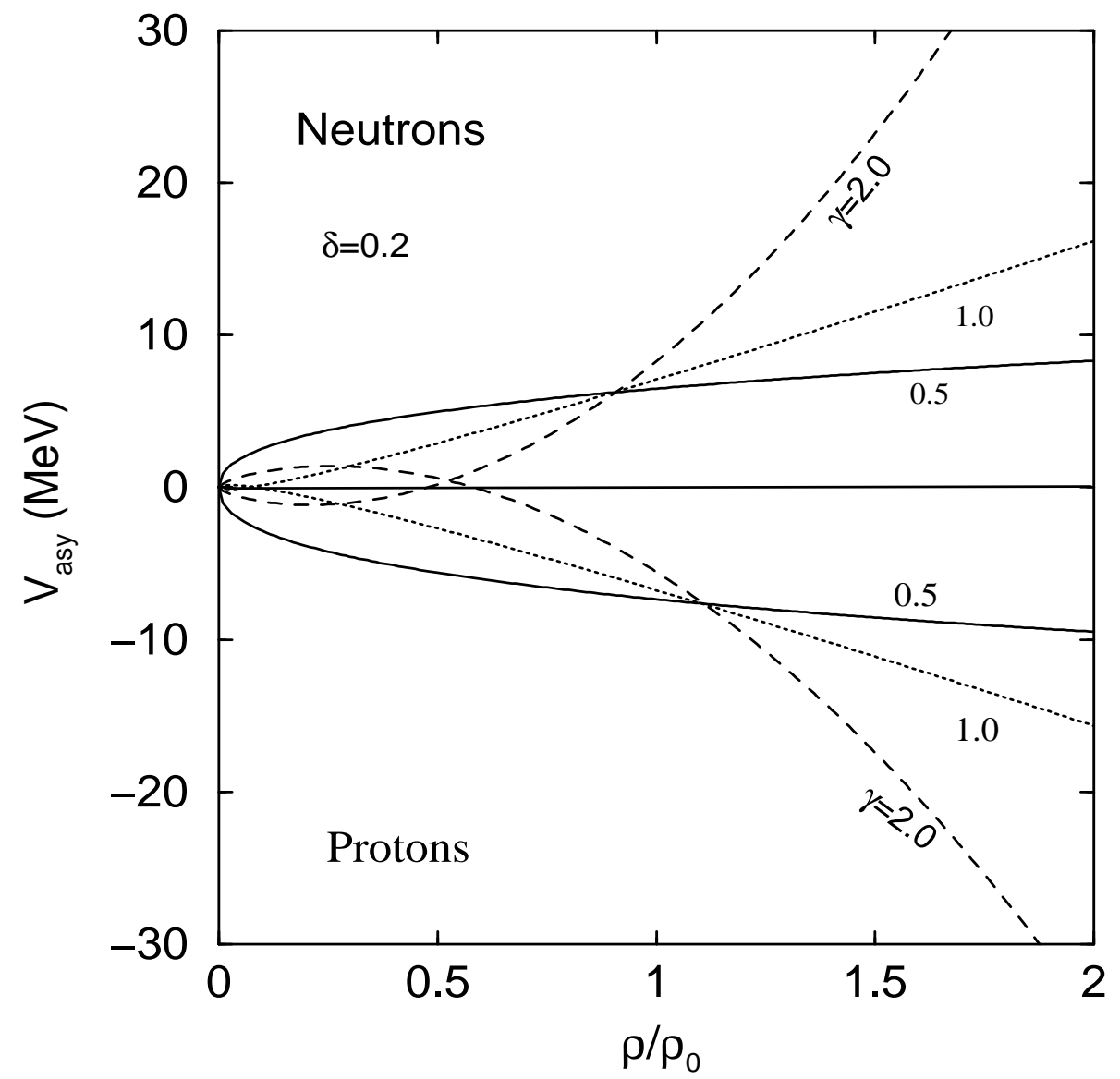

FIG. 1. Symmetry potential for neutrons (upper branches) and protons (lower branches) as a function of density for an isospin asymmetry of $\delta=0.2$ and the $\gamma$ parameter of $0.5,1.0$ and 2.0, respectively. 


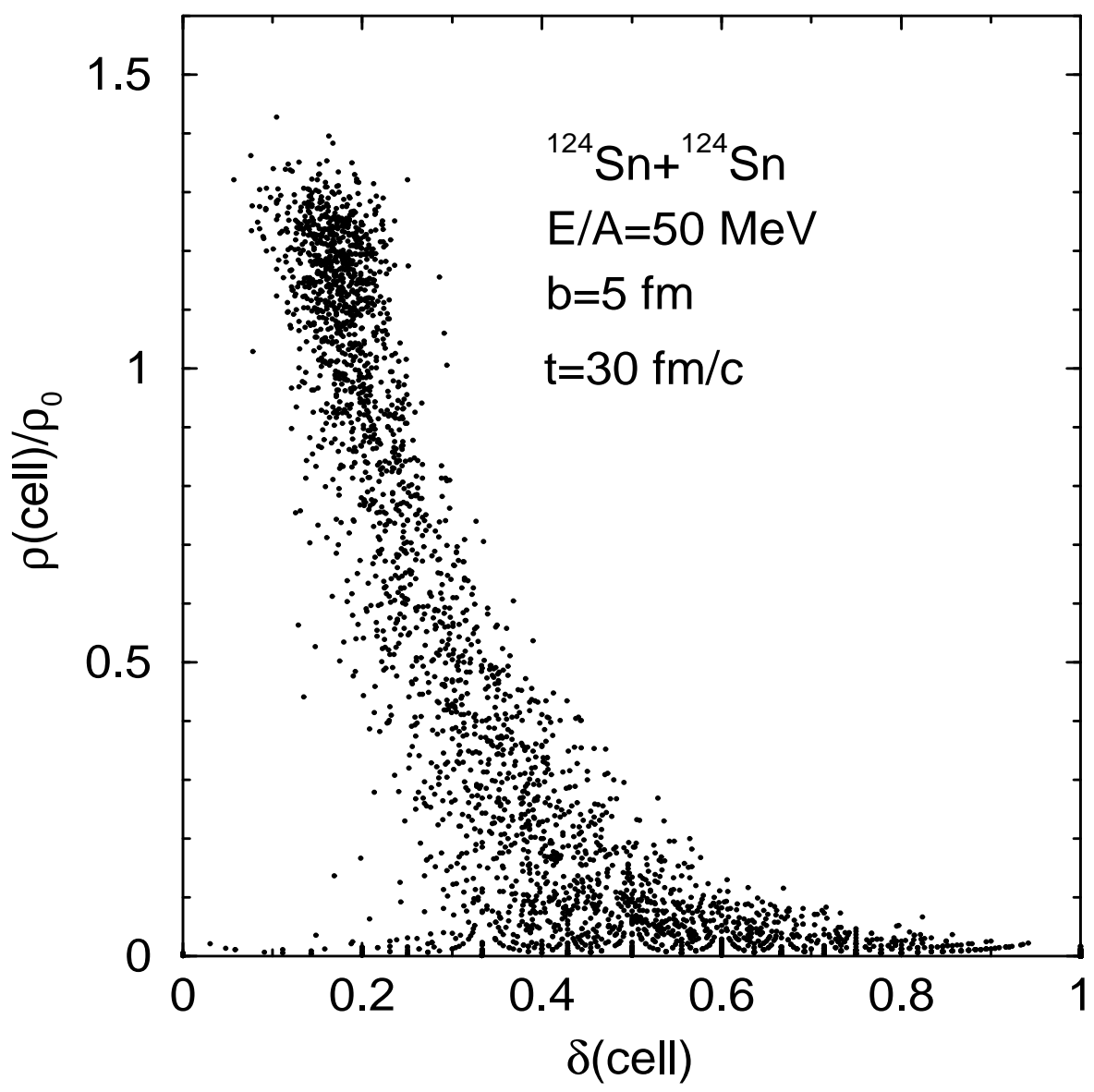

FIG. 2. A scatter plot of the nucleon density of each cell versus its isospin asymmetry $\delta$. 


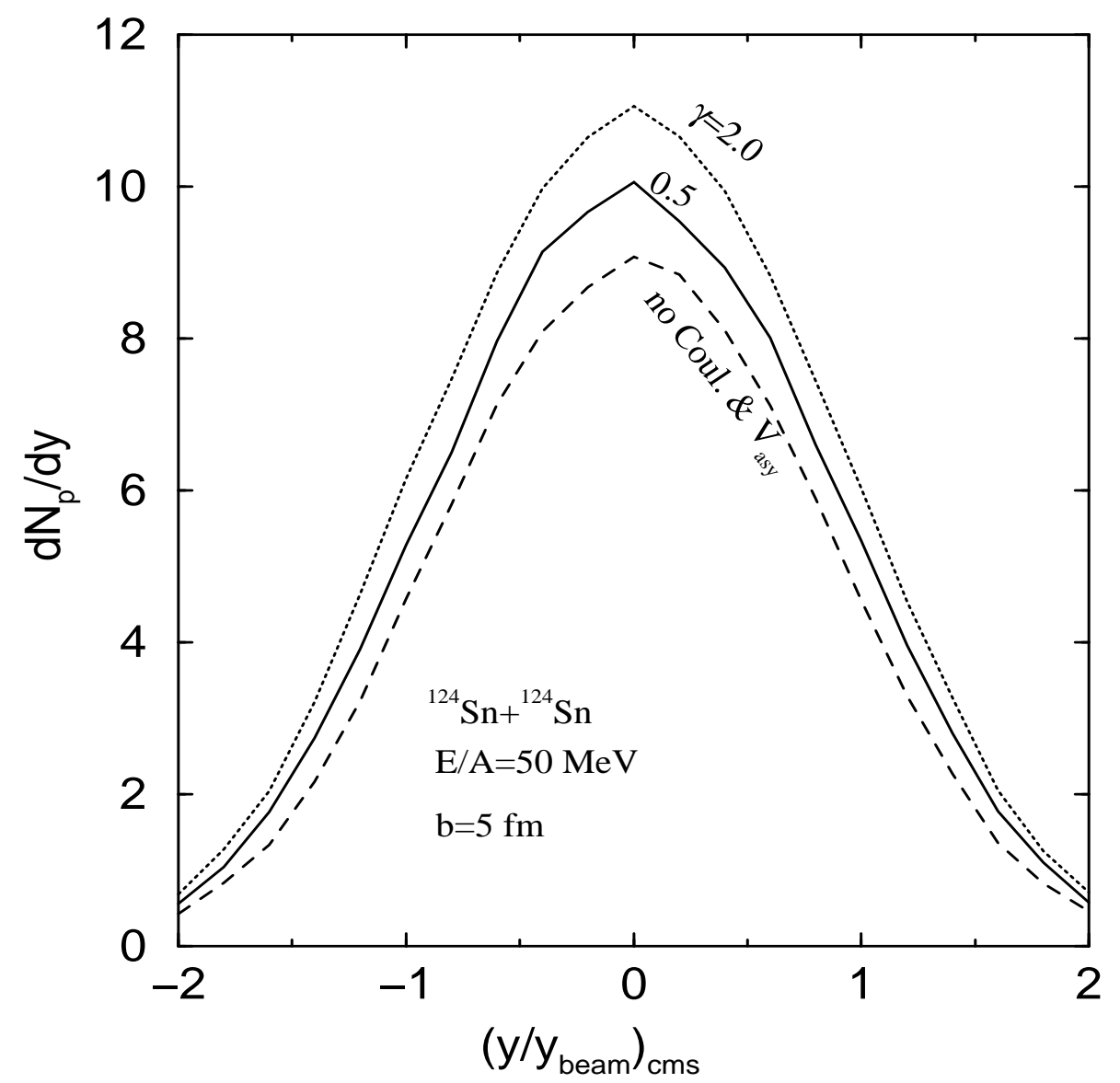

FIG. 3. Rapidity distributions of unbound protons for the $\mathrm{Sn}+\mathrm{Sn}$ reaction. 


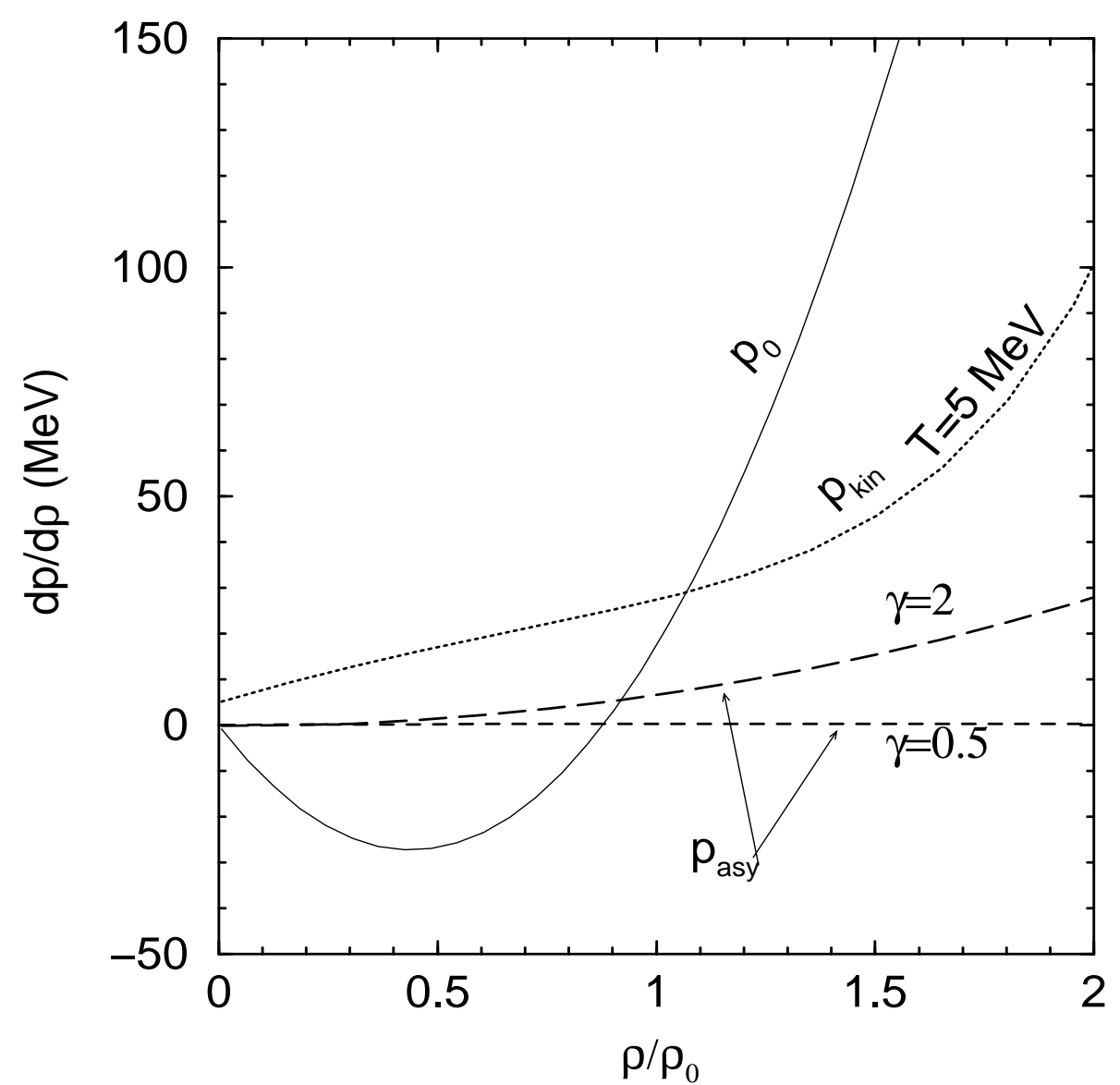

FIG. 4. Derivatives of the three partial pressures as a function of density with the isospin asymmetry $\delta=0.2$ and the $\gamma$ parameter of 0.5 and 2. The solid (dashed and long-dashed) line is for the isospin-independent (dependent) interaction pressure, while the dotted line is for the kinetic pressure at a temperature of $5 \mathrm{MeV}$. 


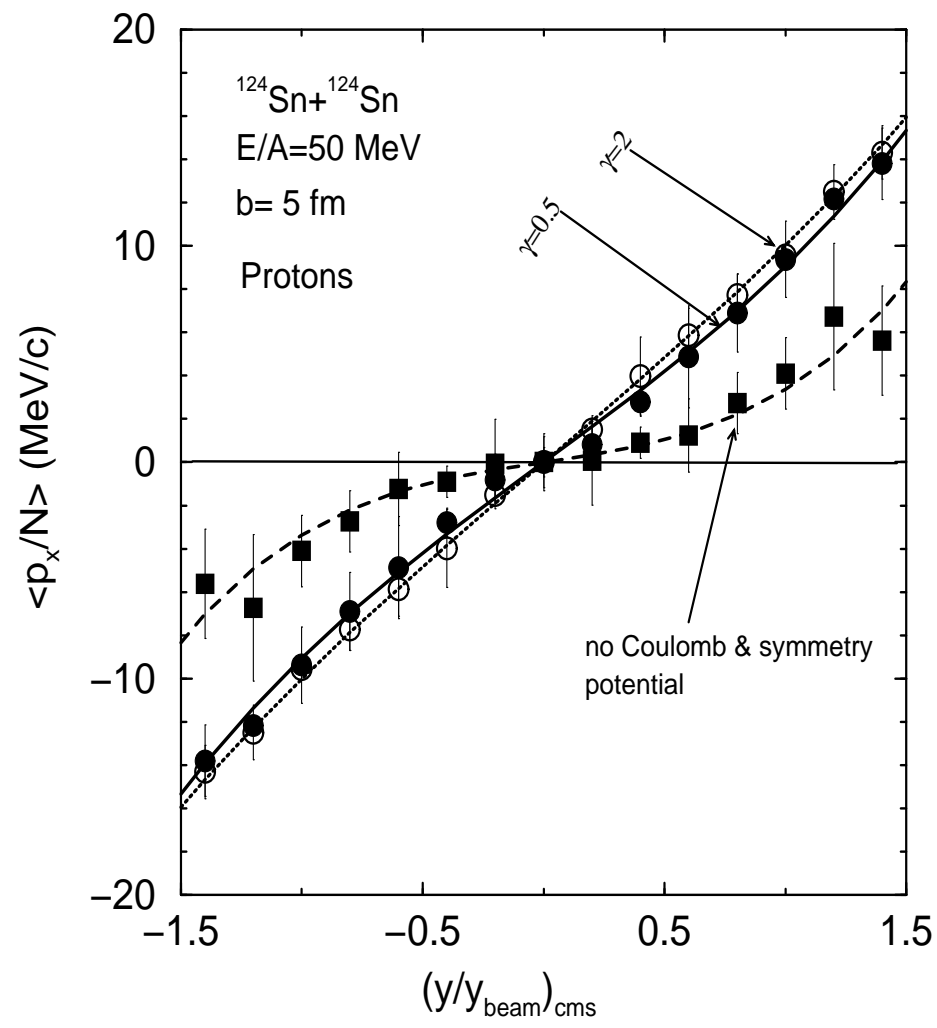

FIG. 5. The average in-plane transverse momentum of free protons as a function of rapidity for the $\mathrm{Sn}+\mathrm{Sn}$ reaction. 


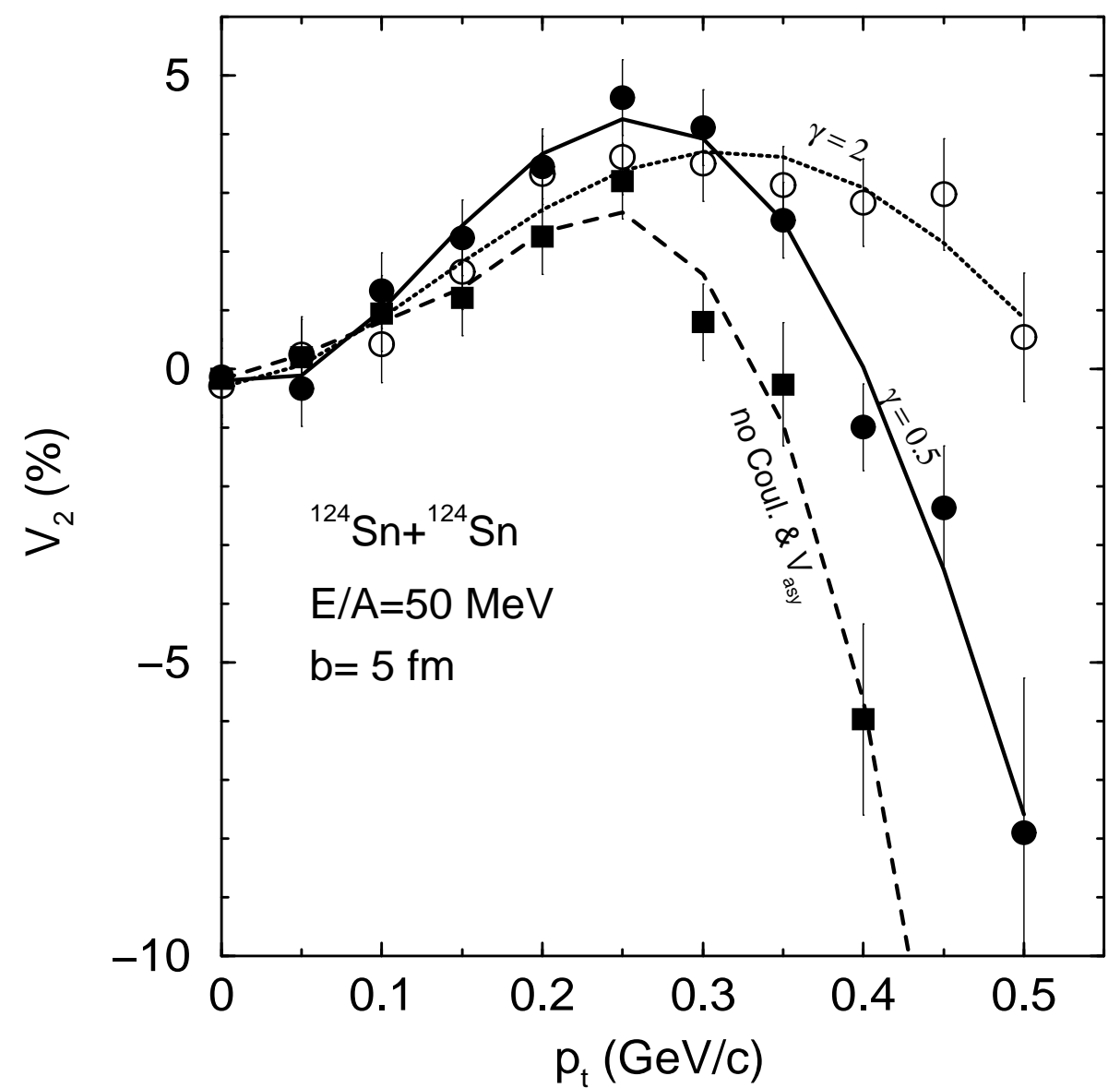

FIG. 6. The elliptic flow for midrapidity free protons as a function of transverse momentum for the $\mathrm{Sn}+\mathrm{Sn}$ reaction. 